\title{
$N 87-17621$
}

GLOBAL DYNAMICS AND THERMAL STRUCTURE OF JUPITER'S ATMOSPHERE

F. M. Flasar

NASA/Goddard Space Flight Center

The presentation by Flasar is largely contained in a paper appearing in the special issue of Icarus (1986; $65,280-303)$. The abstract of his conference presentation is reproduced here:

Jupiter has an intrinsic luminosity, and most, if not all of its interior is believed to be fluid and of low viscosity. These imply a regime of thermally driven turbulent convection. The convection is likely to be strongly constrained by the planet's rotation, and should maintain an adiabatic interior with small horizontal gradients in temperature. Observations at visible wavelengths depict a cloudy atmosphere with a system of westerly and easterly jets with peak-topeak amplitudes of up to $\sim 250 \mathrm{~m} \mathrm{~s}^{-1}$ near the equator. Between the visible cloud layer and the adiabatic interior lies a transition zone about which little is known. Latent heat release by condensibles, disequilibrium between ortho- and para-hydrogen, and baroclinic/ symmetric instabilities might contribute significantly in maintaining the vertical and horizontal thermal stucture of this region. Theory and observation suggest that, except for a limited range of altitudes, the mean lapse rate in the transition zone is close to dry adiabatic. Horizontal gradients in temperature are not well constrained. How the observed multiple jet system is driven is closely related to how deeply it extends into Jupiter; neither is known. A jet system which extends into the adiabatic interior would imply that the convective eddies themselves are providing the required sources of zonal momentum. Analyses of Voyager images have suggested that the eddies at the observable cloud level tend to transport net zonal momentum counter gradiently into the jets, but this interpretation may suffer from nonuniform and incomplete sampling. Moreover, the momentum transport by any secondary circulation induced by such eddies has not been addressed. In the upper troposphere and lower stratosphere, the observed temperatures suggest a decay of the zonal winds with altitude. This is consistent with a forced mean meridional circulation with frictional and radiative damping, which has upwelling and adiabatic cooling at the latitudes of anticyclonic vorticity. In the upper stratosphere, the meridional temperature anomalies reverse sign. The cause of this is not known and could have either a radiative or dynamical origin. Additionally, there is a global hemispheric gradient, suggestive of a seasonal forcing and implying a global cross-equatorial circulation at high altitudes.

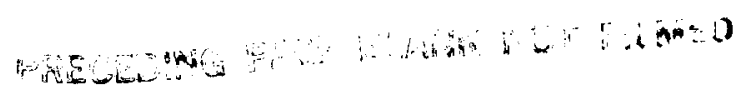


DR. STONE: Would anyone like to make comments or ask questions?

DR. ORTON: Yes, let me ask a question and make a comment in reverse order. First, as I responded yesterday, our monitoring of that strange anomalous area near 24-28 deg $\mathrm{N}$ planetographic was not inconsistent with the area remaining warm over several years, although our resolution was only about $10 \%$ on the disk in ' 81 and changed to $5 \%$ of the disk only later on. On the other hand in 17.8 microns, it seems stable. We did see that the whitening of the equatorial region was almost simultaneous with the cooling off of the central area right at the equator, you notice between '79, ' 80 and ' 81 . What we're doing now is waiting for the South Tropical Zone to unwhiten itself and see what happens therma11y.

DR. FLASAR: You're saying that the temperature decrease is associated with a whitening of this region?

DR. ORTON: Yes, it appears to. Another thing I want to know is about 7.8 microns. Cess et al. report an anti-correlation between the tropospheric and stratospheric temperature peaks, but we find that by 1982 and later and certainly among the maps of 1984, things got terribly screwy and this correlation is obviously too simplistic. Do you have any answers?

DR. FLASAR: No, I had trouble seeing the ones from the Stony Brook slide that was taken at the Voyager time. Some of those correlations--maybe it was just the way it was plotted--were hard for me to pick out. I think the whole business of the albedo changes on the planet is really weird. It might just be a cosmetic effect as far as dynamics goes, but we don't know yet.

DR. HUNTEN: Certainly this is not original with me, but if you're going to correlate A with B then you should plot A against B, not A and B against C.

DR. FELS: There is an analogous situation at several places on the Earth as Conway Leovy, I think it was, first pointed out. In the stratosphere at $50 \mathrm{~km}$ you have the summer poles warm and the winter poles somewhat cooler, whereas in the mesosphere the temperature gradient is reversed and that seems to be the signature of a heating region, a radiative heating region, embedded in a region of strong mechanical friction.

DR. FLASAR: The embarrassing thing is that we already invoked that model at lower altitudes near the tropopause. That's why we need another source of heating in the upper stratosphere.

DR. POLLACK: Mike, you spoke in the beginning part of your talk about counter gradient angular momentum transport by eddies as a way of building up the jets. Would you care to say which eddies are responsible?

DR. FLASAR: One fundamental problem is the relative effect of the eddies we see near the cloud tops, the quasi-geostrophic eddies which are analogous to the kind that have been studied on the Earth, at least at shallow atmospheric levels, and the deeper convective eddies which transport the heat from the interior. That's an open question. 
DR. POLLACK: Do you see shallow eddies as being basically baroclinic?

DR. FLASAR: I don't know. They could be barotropic. Eddies on the scale of the Rossby deformation radius, $\$ 10^{3} \mathrm{~km}$, were not well sampled by the IRIS experiment. If anything, the available data suggest that thermal contrasts are sma11 at these scales.

DR. LIMAYE: You showed some temperature difference from IRIS between Voyager 1 and Voyager 2. What kind of changes in the zonal wind circulation would you see from these temperatures if any?

DR. FLASAR: We11, in a lot of cases the temperature contrast was reduced in Voyager 2 from Voyager 1 , so I guess you would tend to see a weakening of that meridional circulation I talked about. We see very little change in the zonal winds, I think, but Andy or Reta would be better able to address this.

DR. SROMOVSKY: The plots of the vertical wind shear that you showed correlating with $\langle U\rangle$ on Saturn and Jupiter, don't show such a good correlation when you get near the equatorial jet: rather than decaying with height, the shear is building.

DR. FLASAR: I think it's good up to the horns, near 8 deg latitude.

DR. SROMOVSKY: What's the current explanation for the fallure to follow the correlation at very low latitudes?

DR. FLASAR: Well, as you get too low, the thermal wind equation breaks down, although I would expect that to be good to within 3 degrees on Jupiter. Because the Coriolis parameter is small at low latitudes, however, uncertainties in the meridional gradients in temperature translate into large uncertainties in the computed wind shears.

DR. STONE: I'd like to ask a question. You presented this picture of the zonal winds being the balance between the Coriolis force generated by this overturning circulation and some friction. You present that as a purely kinematic picture; you didn't say why it would do that. But let me ask, what value of the frictional time scale do you need in order to explain the balance in that way?

DR. FLASAR: The vertical damping scale of the jets in the lower stratosphere suggests the frictional damping time is roughly comparable with the radiative time constant, about $10^{8} \mathrm{sec}$.

DR. STONE: This whole thing exposes one of the problems in trying to make deductions about the balances there. You're talking about very long forcing and dissipation time scales and that means you're likely to have a very delicate balance and you pointed that out yourself indirectly when talking about the eddies doing sometimes one kind of transport, sometimes another, then also the mean circulations and the eddies being balanced. Just think of the Earth where it took hundreds of stations and many years of observations before we could sort out those balances and here we've got even longer time scales to deal with. 
DR. FLASAR: The situation isn't ideal, but I'm afraid it's all we have.

DR. STONE: Any more questions or comments? Andy Ingersol1?

DR. INGERSOLL: To expand on that, however little you may like the correlation between $u^{\prime}$ and $v^{\prime}$, the amplitude of those eddy winds, just the RMS amplitude, is undeniably large enough so that the time constant for the eddies to destroy a zonal wind is three months. So the eddies have a lot of power there if they should ever get their act together.

DR. FLASAR: There are two points to that. One is that we know that there is an excess of angular momentum, so we know the eddies are getting their act together somewhere. The other is this: if the eddies at the cloud tops are doing nothing or are only tending to erode the jets then it's always possible that if there are eddies deep down in the convective cells, they might get their act together faster and maintain these jets. So there are a lot of "ifs".

DR. EMANUEL: I find it paradoxical that, while we admit to the possibility of deep motions in Jupiter that we talk about this angular momentum budget as though it were two-dimensional on spherical surfaces. Is there any good reason to believe that what we're seeing is not simply the consequence of a vertical transport of angular momentum by the mean motions through horizontal planes? Has this really been ruled out as a possibility? Do we really need eddies to explain this?

DR. FLASAR: I think the theorem I talked about is sufficiently general, you don't have to have shallow planes.

DR. EMANUEL: Of course it's true; to get a real excess of angular momentum that has to be true, but are we seeing a real excess of angular momentum, or are we just seeing what appears to be an excess on a spherical surface? In other words, if we had sinking motion in the equatorial plane that would conserve angular momentum but would appear as an angular acceleration.

DR. FLASAR: That's easy to answer. The excess is about $1 \%$ of the planetary component of angular momentum: $\Omega \mathrm{a}^{2}$. The $\Omega$ comes from System III, which is the radio rotation rate. If that's of $f$, then of course all bets are off; but if you accept that, then it turns out that to get a $1 \%$ excess, you have to have sinking motion from about 350 kilometers above the visible cloud tops which is well above the stratosphere, so I tend to discount that. The thing is, we know superrotation and excess angular momentum occur on other bodies such as Venus where there is no question that it's real. So it kind of buoys up your confidence that it may be occurring on Jupiter also, even though we don't fully understand what is going on.

DR. STONE: I would like to make one more comment myself with regard to the question of whether the forcing is primarily the internal heat source or the solar differential heating. The seasonal variation that Reta Beebe showed us yesterday and the solar differential heating might give us a way of getting at the answer to that question. I didn't really worry too much about seasonal change before, simply because the axis is tilted so little. But Reta showed 
that's too simple minded. Indeed, there does seem to be strong forcing and one should, I think, look for seasonal effects and to the extent that you can find them you know at least that is due to differential solar heaing. You might say that the fact that we haven't seen any strong seasonal changes supports the view that it's the internal heat source that's the more important source. But I think if you go to high enough latitudes at least, you ought to see the effects of the differential heating and it would be very interesting to try to pin down where, if at all, you see those seasonal effects.

DR. FLASAR: That's a good point, and I should also point out that you do have another laboratory that's just a few more A.U. out at Saturn, which is 26 degrees tilted, where the seasonal effects are presumably stronger. So by comparing those two we should get some insight.

DR. STONE: I guess it's time to adjourn for 15 minutes for coffee and more discussion. 